\title{
The Treatment of giant omphalocele by the GROB method (about two cases)
}

\section{Bouanani ibtisseme (university of Sidi-bel-Abbes.ALGERIA)}

\section{Introduction}

The omphalocele, called the middle coelosome, is a congenital malformation due to a lack of plication between the $3 \mathrm{rd}$ and 4 th weeks of amenorrhea.

The curative surgical TRT consists of the closure of the defect and the reintegration of the viscera into the peritoneal cavity. However in the giant forms (type II) according to Aitken this immediate closure is difficult sometimes impossible and burdened with a significant mortality rate.

The non operative and conservative method using wall tanning, which was described by Grob in 1957, was recommended for these forms, or we report our experience and the interest of the latter in the vital prognosis during the neonatal period

\section{Observations}

$\square$ We report 03 cases of giant Omphalocele treated by tanning with $2 \%$ aqueous eosin.

Omphalocele was isolated in 01cas, associated with other malformations in 02 cases.

The consequences of our conservative treatment were favorable with a total epidermis with a decline of 6 months to 1 year. Autoplasty was performed for one patient and the other two were waiting.

Regarding the newborn with poly malformation syndrome, we first treated sacro-coccygeal teratoma followed by tanning, however the infant died following a respiratory infection

\section{Comments}

It is an infrequent congenital malformation predominantly male. Its incidence is 1 case / 4000 birth.

Its prognosis depends on the associated malformations and the size of the collar and its contents.

Recent studies report significantly lower mortality rates than in previous decades

The hyper abdominal pressure caused by immediate closure makes the technique no operative by tanning (Grob).

The tanning is preferably done with $2 \%$ aqueous eosin according to several authors of the same in our experience, which makes it possible to minimize parietal infection, rupture and ileus

\section{conclusion}

The therapeutic approach of giant omphalocele depends on the means and the existing technical platform.

Inadequate conditions of neonatal resuscitation and in which assisted ventilation is not available, as well as the presence of malformations associated with the technique of epidermis by tanning gives good results by considerably reducing the neonatal mortality rate.
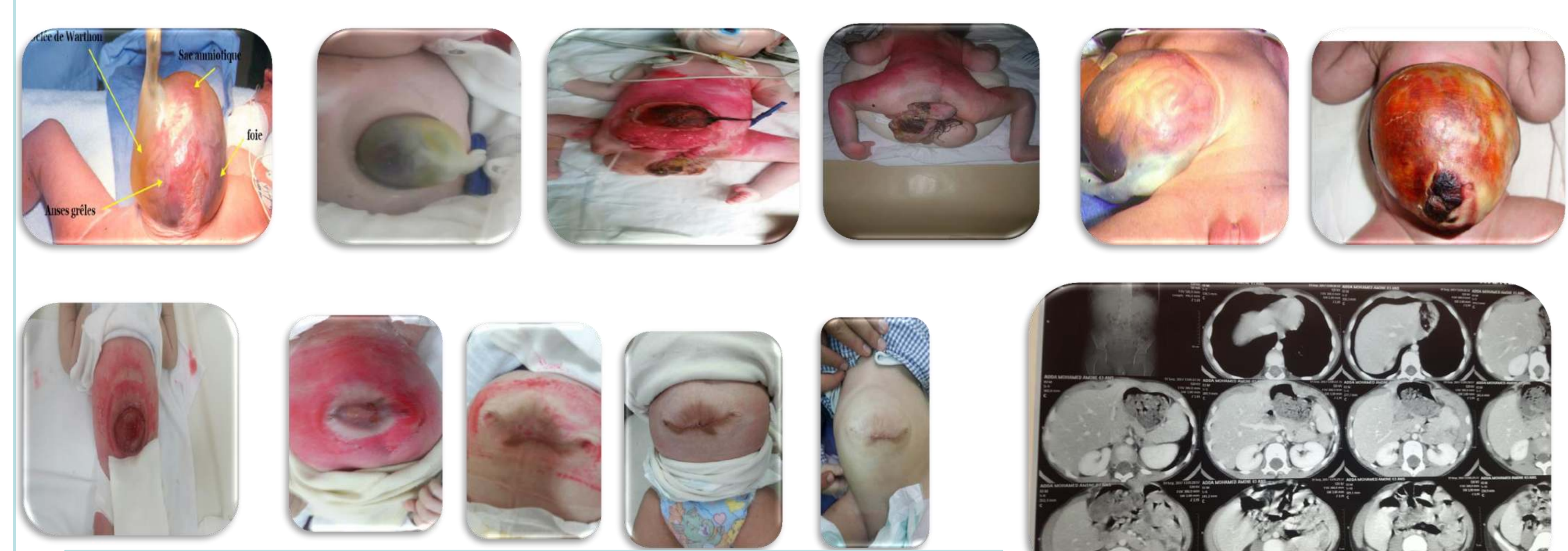

Method of epidermalization by tanning with $2 \%$ aqueous eosin: 1st and 3rd observation (duration 06 months to 1 year)

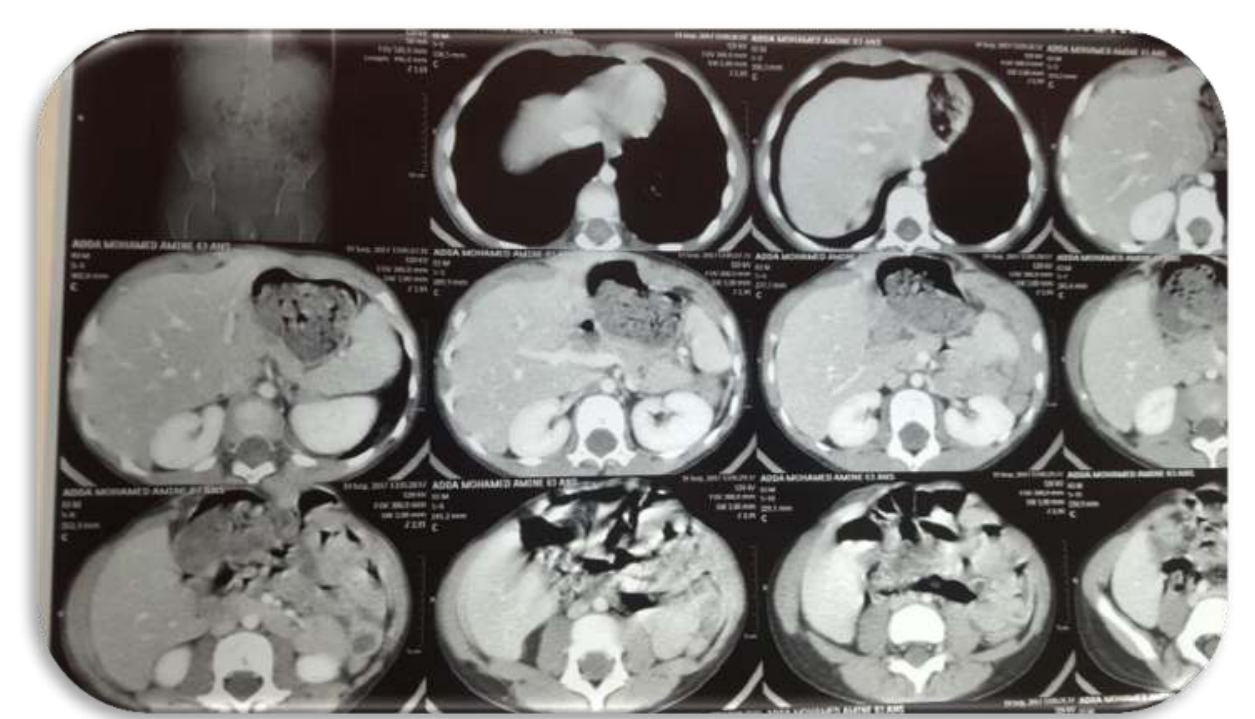

Abdominal scanner : parietal eventration pariétale 